
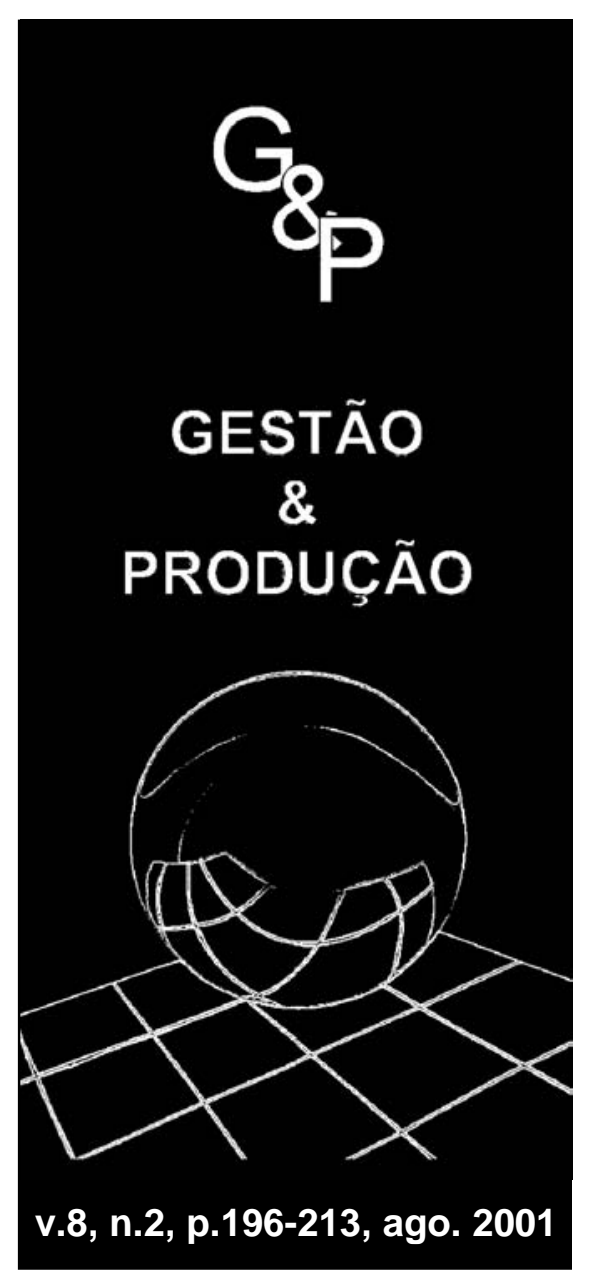

\section{DA ABORDAGEM DO TQM (TOTAL QUALITY MANAGEMENT) AO GQM (GLOBAL QUALITY MANAGEMENT): A INSERÇÃO E UTILIZAÇÃO DA METODOLOGIA DO PROJETO AXIOMÁTICO NO DESENVOLVIMENTO DE MODELOS DE GESTÃO SISTÊMICA DA QUALIDADE}

\author{
Felipe Araújo Calarge \\ Faculdade de Engenharia Mecânica e de Produção \\ Universidade Metodista de Piracicaba \\ E-mail: fcalarge@unimep.br \\ Paulo Corrêa Lima \\ Faculdade de Engenharia Mecânica \\ Universidade Estadual de Campinas \\ E-mail: plima@fem.unicamp.br
}

\title{
Resumo
}

A palavra qualidade tem sido nos últimos anos sinônimo de sucesso ou fracasso em muitas empresas, sendo que esta situação resulta de como estas empresas compreendem os conceitos de qualidade. $O$ desenvolvimento destes conceitos tem feito com que a qualidade deixe de ser simplesmente um meio de controle de produtos e processos, a fim de se desenvolver uma abordagem sistêmica de gestão da qualidade para toda a organização. O objetivo deste artigo é apresentar uma proposta de um modelo de gestão sistêmica da qualidade orientado pelas necessidades e atributos do cliente de uma empresa. No desenvolvimento deste trabalho é utilizado a abordagem do Projeto Axiomático, estabelecida em função de axiomas, corolários e teoremas, com o objetivo de implementar "boas práticas de projeto" na construção do modelo de gestão sistêmica da qualidade.

Palavras-chave: gestão da qualidade, abordagem sistêmica, projeto axiomático, gestão sistêmica da qualidade.

\section{Introdução}

$\mathrm{D}$ entro do contexto impulsionador de transformações em que se inserem a maior parte das indústrias, a palavra "qualidade" tem surgido como um fator capaz de incorporar vantagens competitivas para as organizações, sendo que em nome da qualidade tem-se alterado padrões, exigências, comportamentos sociais e econômicos, criando-se e consolidando-se novos paradigmas dentro do contexto das organizações. Contudo, apesar do inequívoco consenso sobre o 
importante papel desempenhado pelas diferentes abordagens do conceito da qualidade nos dias atuais, uma situação paradoxal é a inexistência de definições consensuais sobre este conceito.

As diferentes abordagens e dimensões que a qualidade pode assumir nas organizações, bem como os estágios evolutivos que o conceito da qualidade vem apresentando, têm implicado na busca de procedimentos que possam sistematizar e gerenciar as questões relativas à qualidade. Dessa forma, como a qualidade poderia ser melhor gerenciada? A qualidade realmente constitui-se em uma vantagem competitiva das organizações? Seria possível avaliar o desempenho da qualidade? É neste contexto que este artigo propõe a discussão destas questões como um dos objetivos principais, abordando aspectos que possibilitem sistematizar de maneira estruturada a construção de um modelo de gestão sistêmica da qualidade, considerando como diretriz principal a abordagem conceituada pelo Total Quality Management (TQM), traduzido neste trabalho como Gestão da Qualidade Total, e contemplando aspectos delineados pelo Global Quality Management (GQM), traduzido aqui como Gestão da Qualidade Global. Para tal, propõem-se a aplicação de uma metodologia de abordagem holística denominada Axiomatic Design, a qual é utilizada para o projeto e construção do modelo de gestão sistêmica da qualidade, tendo sido tal desenvolvimento pautado no que a metodologia denomina de "boas práticas de projeto", estando as mesmas estabelecidas e estruturadas através de axiomas, corolários e teoremas.

\section{Princípios da abordagem do TQM (Total Quality Management)}

$\mathrm{O}$ conceito conhecido e difundido pela sigla TQM (Total Quality Management), ou Gestão da Qualidade Total, tem se consolidado pelo uso extensivo e pela prática das principais abordagens da qualidade, constituindo-se na atualidade em modelos de gerenciamento e administração de questões relativas ao que pode se denominar de qualidade total. As abordagens clássicas estabelecidas pelos "gurus" da qualidade (Feigenbaum, Deming, Juran, Ishikawa, Taguchi e Crosby) dentre outros, são visualizadas dentro da abordagem do TQM por alguns autores como “... falando um mesmo idioma através de diferentes dialetos, tendo princípios comuns tanto ao definir a qualidade como ao considerá-la através de todas as atividades da empresa..." (OAKLAND, 1994).

O papel integrador de todos os aspectos da qualidade que envolvam pessoas, equipamentos, recursos computacionais e estrutura organizacional, classificam o TQM como um conceito para se atingir abordagens sistêmicas para a gestão da qualidade (BADIRU \& AYENI, 1994). Outro papel fundamental atribuído ao TQM envolve o seu relacionamento com a busca constante da satisfação do cliente, pela utilização integrada de ferramentas e técnicas que envolvam a melhoria contínua dos processos organizacionais (SASHKIN \& KISER, 1994). Uma abordagem baseada em uma visão acentuadamente holística qualifica o TQM como agente modificador de valores e práticas sociais, nos quais paradigmas estariam sendo firmados em função não do estabelecimento dos recursos de uma organização como recursos próprios, mas sim como recursos globais (MOHANTY, 1997).

Dados os exemplos citados que descrevem os diferentes enfoques pelo qual o TQM pode ser visualizado, verifica-se que apesar do consenso existente sobre a importância em relação à qualidade total, existem diferentes maneiras ou mecanismos pelo qual uma organização pode atingi-la. Neste sentido, descrevendo alguns modelos e propostas metodológicas citados pela literatura, pode-se relacionar a proposta de OAKLAND (1994), a qual aborda que o núcleo do TQM deve estar fundamentado na relação cliente-fornecedor, tanto internos quanto externos à organização, definindo e associando processos de conversão de inputs em outputs. Segundo LAKHE \& MOHANTY (1994) outros 03 modelos esquemáticos do TQM poderiam ser citados: 
- modelo tridimensional proposto por Price \& Gaskill atribui 03 dimensões à qualidade: dimensão do produto e serviço, dimensão de pessoas e dimensão do processo. Este modelo aborda basicamente o grau de satisfação do cliente com relação a produto e/ou serviço; grau de satisfação existente entre as pessoas envolvidas na relação cliente-fornecedor e o grau de satisfação do cliente com os processos internos do fornecedor.

- modelo integrado proposto por Sohal, Tay e Wirth, o qual aborda que a melhoria contínua em qualidade advém de uma abordagem integrada de controlar a qualidade via planos de ações táticas em diferentes operações durante o ciclo de negócio, definindo 05 principais elementos: foco no cliente, comprometimento da gerência, participação total, controle estatístico da qualidade e foco na solução sistemática de problemas.

- modelo de "blocos de construção" proposto por Zaire, o qual estabelece que o TQM depende de fases construtivas que determinam a rigidez e segurança da organização. Assim, estabelece-se como fases construtivas a fundação, pilares e topo, sendo que a cada fase são definidas ações e elementos necessários para sua construção.

Contudo, um assunto que tem mobilizado esforços das mais diversas áreas acadêmicas e empresariais, é a questão se o TQM poderia agir como uma fonte potencial de vantagem competitiva. A teoria da Visão Baseada em Recursos (RBV - Resources Based View) apresenta uma perspectiva destas questões, iniciando com a noção da heterogeneidade de recursos, ou seja, diferentes firmas possuem diferentes portfólios de recursos de maneira que tais diferenças podem induzir a uma variabilidade no desempenho entre estas firmas.

Dessa forma, poderia o TQM ser considerado um recurso? E se assim o fosse, poderia ser assimilável entre as empresas, produzindo valor para as firmas que o implantassem? Estas questões tem gerado controvérsias, sendo que o questionamento sobre a criação de valor pelo
TQM esbarraria no que se denomina de imitabilidade imperfeita, ou seja, a difusão de inovações daria-se entre firmas que possuem considerável grau de similaridade (denominadas de firmas homófilas). Nesta situação, considerando o TQM como uma forma de inovação da empresa, tanto a empresa inovadora como uma potencial empresa adotante da inovação deveriam ter de compartilhar similares atributos relativos a objetivos, estratégias, normas, crenças, experiências e culturas (POWELL, 1995).

\section{Princípios da abordagem do GQM (Global Quality Management)}

I ndependentemente do TQM ter sido adotado por muitas empresas com consideráveis efeitos positivos, as questões colocadas em muitas economias atuais dizem respeito à denominada globalização de mercados e as conseqüências estruturais destas mudanças. Um número cada vez maior de empresas freqüentemente tem-se deparado com diversidades de necessidades requeridas pelos mercados em que atuam, as quais podem envolver aspectos que abrangem desde localizações geográficas até hábitos e costumes culturais distintos. Neste contexto, poderia a motivação e o envolvimento pela qualidade afetar todos os níveis e funções de uma organização estruturada para atuar em diferentes mercados globalizados? Esta questão ainda tem suscitado um número maior de indagações do que de respostas definitivas.

Contudo, uma linha de pesquisa que tem se aprofundado em tais questões, discutindo o gerenciamento da qualidade em organizações globalizadas, é o que se denomina de $G Q M$ (Global Quality Management), ou "Gestão da Qualidade Global", que segundo KIM \& CHANG (1995) seria definida como “... um planejamento estratégico com interação de produtos e processos, para que se obtenha alta aceitação do cliente e baixa disfuncionalidade organizacional através de mercados em diferentes países". Esta visão incorpora a filosofia central básica do $\mathrm{TQM}$, reconhecendo contudo as diferenças no 
ambiente de qualidade através dos mercados globais, implicando assim na necessidade de balanceamento dos requisitos locais dos clientes com as capacidades de redes de fornecimento das organizações. KIM \& CHANG (1995) acrescentam ainda que a evolução do conceito da qualidade em direção ao GQM estaria estruturada ao longo de 05 dimensões básicas que seriam:

a) Escopo: expande o conceito de qualidade de maneira a contemplá-la em múltiplas funções através de diferentes países englobando aspectos que abordariam soberanias nacionais; distinções culturais e de língua; aspectos geográficos; considerações tarifárias e fiscais; etc.

b) Orientação de Mercado: dada a dificuldade de se contemplar todas as diversidades exigidas pelos clientes no sentido de satisfação de suas necessidades, deve-se ir ao encontro das percepções de valores dos clientes, devendo para isto ter-se uma exata compreensão das particularidades e sensibilidades culturais dos mercados em que a organização busca atuar.

c) Orientação da Produção: busca-se a extensão ao ponto do aparecimento de disfuncionalidades, envolvendo todas as funções e todos os níveis, em todos os países. A situaçãochave que possibilitaria às empresas minimizarem suas potenciais disfuncionalidades em um contexto globalizado seria a flexibilidade, no sentido da busca simultânea da produção de uma alta variedade de produtos com baixo volume e baixo custo.

d) Sistema de Informação: busca-se instrumentalizar as redes de informações entre as unidades fabris, de modo que a informação possa fluir através da organização global, buscando um efeito sinergético na utilização da informação.

e) Rede Tecnológica: deve-se estar preparado para situações competitivas entre empresas que envolvam diferentes bases tecnológicas, redes de fornecedores, sistemas de produção, atividades de distribuição e atividades de marketing em diferentes países, buscando o estabelecimento de alianças estratégicas que possibilitem a empresas parceiras compartilhar recursos complementares, ressaltando que tais tipos de parcerias estratégicas devem contemplar a compatibilidade de sistemas de qualidade interempresas.

Outro aspecto relevante aborda a métrica padronizadora imposta pelas certificações dos sistemas da qualidade, que dentro do contexto do GQM poderiam não ser adequadas dependendo do grau de diferença de características, as quais podem captar distintas dimensões da qualidade. No entanto, apesar do GQM colocar-se talvez como uma nova questão paradigmática com relação ao escopo da qualidade em mercados globalizados, muito ainda tem que ser desenvolvido e pesquisado para que se passe das premissas para as constatações. A Tabela 1 indica três estágios evolutivos da qualidade em busca do GQM, indicando características relativas a cada dimensão segundo o respectivo estágio da qualidade.

\section{Conceitos e fundamentos principais do Projeto Axiomático}

A abordagem do Projeto Axiomático constitui-se basicamente de axiomas, teoremas, corolários e suas aplicações no sentido de estabelecer o que se poderia considerar como "boas bases e princípios" de um projeto, sendo a descrição desta metodologia estabelecida em função do propositor do Axiomatic Design, Prof. Nam P. Suh, em sua obra principal neste assunto intitulada The Principles of Design (SUH, 1990). Nesta abordagem, o ato de projetar envolve um contínuo relacionamento entre "qual o objetivo que se deseja atingir" e "como se pode atingir tal objetivo", verificando que os objetivos de um projeto colocam-se dentro do que se denomina de domínio funcional, ao passo que as soluções encontradas para atingir os objetivos são sempre geradas no que se denomina de domínio físico. Estes dois domínios são independentes e se inter-relacionam constantemente dentro das atividades de projeto através do estabelecimento de uma estrutura hierárquica 
Tabela 1 - Evolução dos conceitos da qualidade em busca do GQM Fonte: KIM \& CHANG, 1995

\begin{tabular}{|c|c|c|c|}
\hline & $\begin{array}{c}\text { ESTÁGIO I } \\
\text { Controle Estatístico } \\
\text { da Qualidade }\end{array}$ & $\begin{array}{c}\text { ESTÁGIO II } \\
\text { Gestão da Qualidade } \\
\text { Total } \\
\end{array}$ & $\begin{array}{c}\text { ESTÁGIO III } \\
\begin{array}{c}\text { Gestão da Qualidade } \\
\text { Global }\end{array} \\
\end{array}$ \\
\hline ESCOPO & $\begin{array}{l}\text { Baseado no produto e } \\
\text { funcional }\end{array}$ & $\begin{array}{l}\text { Organização } \\
\text { interfuncional }\end{array}$ & $\begin{array}{l}\text { Interorganizacional e } \\
\text { Internacional }\end{array}$ \\
\hline $\begin{array}{l}\text { ORIENTACÃO DE MERCADO } \\
\text { - Enfoque de valor com relação } \\
\text { ao consumidor } \\
\text { - Sensibilidade à Cultura }\end{array}$ & $\begin{array}{l}\Rightarrow \text { Baixa Prioridade } \\
\Rightarrow \text { Baixa }\end{array}$ & $\begin{array}{l}\Rightarrow \text { Alta Prioridade } \\
\Rightarrow \text { Moderada }\end{array}$ & $\begin{array}{l}\Rightarrow \text { Alta Prioridade } \\
\text { mas Diversificado } \\
\Rightarrow \text { Alta }\end{array}$ \\
\hline $\begin{array}{l}\text { ORIENTACÃO DE PRODUCC̃̃O } \\
\text { - Sistemas de Operação } \\
\text { - Localização do Controle } \\
\text { - Flexibilidade }\end{array}$ & 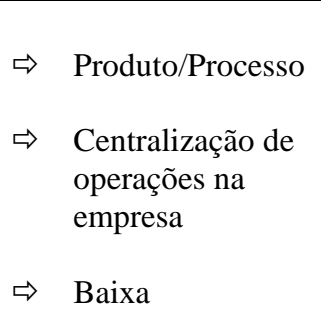 & $\begin{array}{l}\Rightarrow \text { Organização } \\
\Rightarrow \quad \begin{array}{l}\text { Coordenação de } \\
\text { operações pela } \\
\text { empresa }\end{array} \\
\Rightarrow \text { Moderada }\end{array}$ & $\begin{aligned} \Rightarrow & \text { Organização } \\
& \text { interpaíses } \\
\Rightarrow & \text { Múltiplas } \\
& \text { coordenações em } \\
& \text { uma rede global de } \\
& \text { operações } \\
\Rightarrow & \text { Alta }\end{aligned}$ \\
\hline SISTEMAS DE INFORMACÃO & $\begin{array}{l}\text { Localizado a nível da } \\
\text { planta fabril }\end{array}$ & Integrado à corporação & Integrado globalmente \\
\hline REDE TECNOLÓGICA & $\begin{array}{l}\text { Localizado a nível da } \\
\text { planta fabril }\end{array}$ & Rede corporativa & $\begin{array}{l}\text { Rede tecno-econômica } \\
\text { global }\end{array}$ \\
\hline
\end{tabular}

nestes relacionamentos, cujo resultado final desta interação entre domínios é o próprio projeto, ou seja, soluções encontradas para os objetivos que foram colocados.

Deve-se então, determinar objetivos do projeto definindo-os em termos de necessidades específicas, as quais serão denominadas de Requisitos Funcionais, sendo indicadas neste trabalho pela sigla FR (Functional Requirement), optando-se por manter neste trabalho a nomenclatura já amplamente difundida por Suh. Para satisfazer tais requisitos funcionais, entidades físicas devem ser criadas sendo as mesmas denominadas de Parâmetros de Projeto, indicadas neste trabalho por DP (Design Parameters), mantendo-se a mesma proposição já mencionada anteriormente. Considerando uma visão mais ampliada, na abordagem do Axiomatic Design o ato de projetar pode ser composto em quatro domínios: o domínio do cliente; o domínio funcional; o domínio físico e o domínio de processo. Durante esta fase de projeto, o domínio do cliente deve ser o primeiro a ser explorado a fim de se determinar os atributos do cliente (CAs) para o projeto. Tais atributos devem ser interpretados então como requisitos funcionais (FRs) no domínio funcional que, por sua vez, devem ser satisfeitos em função da escolha de um conjunto adequado de parâmetros de projeto (DPs) no domínio físico. Finalmente, no domínio de processo um conjunto adequado de variáveis de processo (PVs) deve ser definido. A Figura 1 esquematiza esta situação de interação entre os domínios citados.

Teria-se a seguinte situação no caso do projeto:

- o domínio do cliente consiste nas necessidades ou atributos que o cliente procura em um produto.

- o domínio funcional consiste nos requisitos funcionais de um produto, também denominados de especificações de engenharia.

- o domínio físico é o domínio no qual os específicos parâmetros de projeto (DPs) são escolhidos de modo que satisfaçam os referidos requisitos funcionais (FRs). 


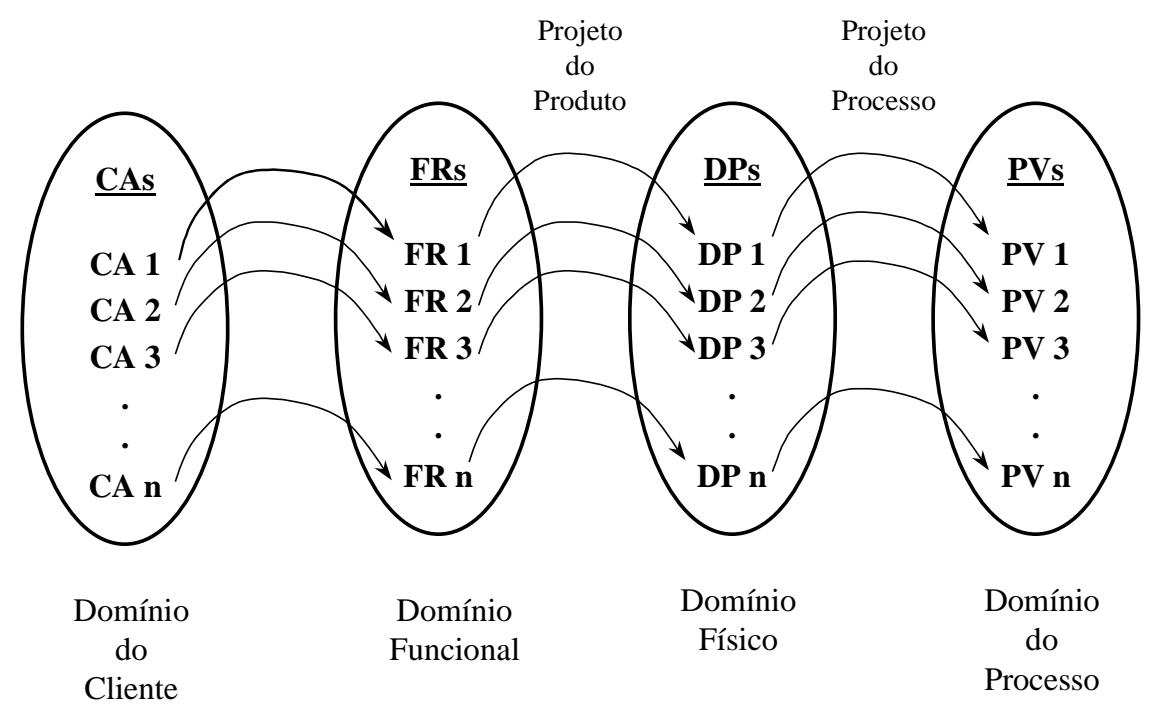

Figura 1 - Domínios existentes na visão ampliada do projeto Fonte: SUH, 1995

- domínio de processo especifica os métodos de manufatura capazes de produzir os parâmetros de projeto determinados.

Dentro desta abordagem, pode-se caracterizar um projeto como uma criação de soluções sintetizadas para requisitos solicitados, podendo tais soluções serem concretizadas na forma de produtos, processos, sistemas ou estruturas organizacionais, existindo então inúmeras soluções de projeto plausíveis e diferentes técnicas de relacionamentos, os quais dependem de fatores relacionados à criatividade e experiência do projetista. O Projeto Axiomático provê os princípios que devem nortear a execução de boas práticas de projeto, oferecendo bases comparativas e seletivas entre as possibilidades existentes.

Quando da elaboração de um projeto, dois fatores importantes devem ser ressaltados dentro da abordagem do Projeto Axiomático:

- O primeiro é que os requisitos funcionais e os parâmetros de projetos possuem níveis de hierarquização e podem ser decompostos.

- O segundo é que os requisitos funcionais em um determinado nível da estrutura hierárquica não podem ser decompostos em níveis subseqüentes na hierarquia de requisitos funcionais sem que antes sejam desenvolvidos os cor- respondentes parâmetros de projeto dentro da hierarquia de parâmetros de projeto.

Essas duas estruturações hierárquicas relacionam-se entre si num processo contínuo de busca e proposição de soluções (denominado de processo zig zag), fazendo com que ocorra a decomposição em níveis hierárquicos subseqüentes da estrutura. Esta possibilidade de "cruzamento" entre as duas estruturas hierárquicas de domínios reduz a complexidade do projeto, pois pode-se restringir a um número limitado de FRs e DPs a cada nível hierárquico da estrutura. No processo de zig zag da estrutura hierárquica de relacionamentos de requisitos funcionais e parâmetros de projeto, FRs e DPs em níveis superiores da estrutura podem restringir soluções em níveis inferiores da estrutura.

Dois axiomas diretivos norteiam a abordagem axiomática, sendo eles:

- Axioma 1 - Axioma da Independência: define que o relacionamento de FRs no domínio funcional com DPs no domínio físico deve ser tal que a interferência em determinado DP deve afetar somente o referente FR, mantendo assim a independência 
dos requisitos funcionais. Deve-se observar duas declarações importantes: um projeto ótimo deve manter a independência de FRs e em um projeto aceitável DPs e FRs se relacionam de forma tal que, determinado DP pode ser ajustado para satisfazer seu correspondente FR sem que afete outros requisitos funcionais.

Este processo de relacionamento de FRs no domínio funcional com DPs no domínio físico pode ser representado matematicamente, desde que as características necessárias ao projeto possam ser representadas por um conjunto de independentes FRs tratados como um vetor FR, o qual possui um número específico de componentes. De forma similar, DPs no domínio físico também podem constituir um vetor DP com um número específico de componentes. $\mathrm{O}$ ato de projetar envolveria então selecionar o conjunto adequado de DPs que satisfizessem a um dado conjunto de FRs, podendo ser expresso da seguinte forma:

$$
\{\mathrm{FR}\}=[\mathrm{A}]\{\mathrm{DP}\}
$$

(Equação 1)

onde:

$\{$ FR $\}$ : vetor de requisitos funcionais

$\{\mathrm{DP}\}$ : vetor de parâmetros de projeto

[A ] : matriz de projeto

Cada linha de vetor da equação (1), a qual será denominada de equação de projeto, poderia então ser descrita como:

$$
\mathrm{FR}_{\mathrm{i}}=\sum_{\mathrm{j}} \mathrm{A}_{\mathrm{ij}} \mathrm{DP}_{\mathrm{j}}
$$

(Equação 2)

A matriz de projeto [ A ] seria da forma:

$$
[\mathrm{A}]=\left[\begin{array}{ccccc}
\mathrm{A}_{11} & \mathrm{~A}_{12} & \cdot & . & \mathrm{A}_{1 \mathrm{n}} \\
\mathrm{A}_{21} & \mathrm{~A}_{22} & \cdot & \cdot & \mathrm{A}_{2 \mathrm{n}} \\
\cdot & \cdot & \cdot & \cdot & \cdot \\
\cdot & \cdot & \cdot & \cdot & \cdot \\
\mathrm{A}_{\mathrm{m} 1} & \mathrm{~A}_{22} & \cdot & \cdot & \mathrm{A}_{\mathrm{mn}}
\end{array}\right]
$$

(Equação 3)

Cada elemento $\mathrm{A}_{\mathrm{ij}}$ da matriz refere-se a um componente do vetor FR para um dado componente do vetor DP, sendo que quando na equação (3) m for igual a n, [ A ] é uma matriz quadrada. Para exemplificar, sendo $\mathrm{m}=\mathrm{n}=\mathrm{k}$ a matriz de projeto seria dada por:

$$
[\mathrm{A}]=\left[\begin{array}{ccccc}
\mathrm{A}_{11} & \mathrm{~A}_{12} & \cdot & \cdot & \mathrm{A}_{1 \mathrm{k}} \\
\mathrm{A}_{21} & \mathrm{~A}_{22} & \cdot & \cdot & \mathrm{A}_{2 k} \\
\cdot & \cdot & \cdot & \cdot & \cdot \\
\cdot & \cdot & \cdot & \cdot & \cdot \\
\mathrm{A}_{k 1} & \mathrm{~A}_{k 2} & \cdot & \cdot & \mathrm{A}_{k \mathrm{k}}
\end{array}\right]
$$

O caso mais simples de um projeto ocorre quando todos os elementos não diagonais são iguais a zero e neste caso a equação de projeto seria expressa por:

$$
\begin{aligned}
\mathrm{FR}_{1} & =\mathrm{A}_{11} \mathrm{DP}_{1} \\
\mathrm{FR}_{2}= & \mathrm{A}_{22} \mathrm{DP}_{2} \\
& \cdot \\
\mathrm{FR}_{\mathrm{k}}= & \mathrm{A}_{\mathrm{kk}} \mathrm{DP}_{\mathrm{k}}
\end{aligned}
$$

Um projeto que pudesse ser representado pela equação (5) satisfaz o axioma 1 , pois a independência de FRs é assegurada quando DPs são alterados, podendo o projeto ser representado por uma matriz diagonal cujos elementos diagonais são os únicos diferentes de zero, sendo este projeto denominado de uncoupled. Aqui cabe a observação que dada a terminologia definida por SUH (1990) ser amplamente difundida e empregada pelos utilizadores da abordagem axiomática, bem como a inexistência de um consenso na tradução adequada para a língua portuguesa, este artigo manterá o termo original em inglês.

$\mathrm{O}$ oposto de um projeto uncoupled seria o que se denomina de projeto coupled, no qual a matriz de projeto constituiria-se de elementos diferentes de zero, tendo neste caso a equação de projeto a seguinte configuração:

$$
\begin{aligned}
\mathrm{FR}_{1}= & \mathrm{A}_{11} \mathrm{DP}_{1}+\mathrm{A}_{12} \mathrm{DP}_{2}+\ldots+\mathrm{A}_{1 \mathrm{k}} \mathrm{DP}_{\mathrm{k}} \\
\mathrm{FR}_{2}= & \mathrm{A}_{21} \mathrm{DP}_{1}+\mathrm{A}_{22} \mathrm{DP}_{2}+\ldots+\mathrm{A}_{2 \mathrm{k}} \mathrm{DP}_{\mathrm{k}} \\
& \cdot \\
\mathrm{FR}_{\mathrm{k}}= & \mathrm{A}_{\mathrm{k} 1} \mathrm{DP}_{1}+\mathrm{A}_{\mathrm{k} 2} \mathrm{DP}_{2}+\ldots+\mathrm{A}_{\mathrm{kk}} \mathrm{DP}_{\mathrm{k}}
\end{aligned}
$$

(Equação 6) 
Tomando-se como exemplo esta equação (6), pode-se verificar no projeto coupled que uma alteração em $\mathrm{FR}_{1}$ não poderia ser feita somente alterando-se $\mathrm{DP}_{1}$, pois isto também afetaria

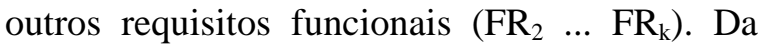
mesma forma isto se daria para os outros requisitos funcionais, sendo que esta situação violaria o axioma 1.

Um terceiro caso denominado de projeto decoupled, tem-se a matriz de projeto [A] triangular, sendo a equação de projeto definida por:

$$
\left\{\begin{array}{c}
\mathrm{FR}_{1} \\
\mathrm{FR}_{2} \\
\cdot \\
\cdot \\
\mathrm{FR}_{k}
\end{array}\right\}=\left[\begin{array}{ccccc}
\mathrm{A}_{11} & 0 & \cdot & \cdot & 0 \\
\mathrm{~A}_{21} & \mathrm{~A}_{22} & \cdot & \cdot & 0 \\
\cdot & \cdot & & \cdot \\
\cdot & \cdot & & \cdot \\
\mathrm{A}_{\mathrm{k} 1} & \mathrm{~A}_{\mathrm{k} 2} & \cdot & \cdot & \mathrm{A}_{\mathrm{kk}}
\end{array}\right]\left\{\begin{array}{c}
\mathrm{DP}_{1} \\
\mathrm{DP}_{2} \\
\cdot \\
\cdot \\
\mathrm{DP}_{k}
\end{array}\right\}
$$

(Equação 7)

Neste caso do projeto decoupled, a independência de FRs pode ser assegurada desde que o ajuste de DPs seja feito de maneira ordenada, ou seja, a seqüência de alteração de DPs é a chave para se garantir a independência de FRs, mantendo-se assim o axioma 1. Exemplificando para a equação (7), se $\mathrm{DP}_{1}$ for alterado primeiramente pode-se ajustar $\mathrm{FR}_{1}$. Embora isto possa afetar outros requisitos funcionais $\left(\mathrm{FR}_{2} \ldots \mathrm{FR}_{\mathrm{k}}\right)$, os parâmetros de projeto $\left(\mathrm{DP}_{2} \ldots \mathrm{DP}_{\mathrm{k}}\right)$ podem ser ajustados seqüencialmente sem que se altere $o$ valor dos requisitos funcionais anteriores.

- Axioma 2 - Axioma da Informação: refere-se à minimização do conjunto de informações, estabelecendo que dentre vários projetos que satisfaçam o axioma 1, aquele que se processa com um menor conjunto de informações é o melhor.

Dentro da abordagem do Axiomatic Design a discussão do conceito de informação é feita sob a premissa da mesma estar relacionada com a probabilidade de satisfazer os requisitos funcionais, de forma que quanto maior for a probabilidade de se satisfazer um requisito funcional, menor será o conteúdo de informação necessário. Sob esta ótica, o axioma da informação pode matematicamente ser representado como segue:

$$
\mathrm{I}=\log _{2}(1 / \mathrm{p}) \text {, onde: }
$$

$I$ = conteúdo de informação

$\mathrm{p}=$ probabilidade de se satisfazer uma necessidade específica

Apesar de reconhecer a importância e relevância que a gestão da informação exerce nas organizações atuais, a diretriz principal deste artigo dada a limitações inerentes ao total de conteúdo abordado, estará pautada na análise do axioma 1 .

\section{Uma proposta de desenvolvimento de um modelo de gestão sistêmica da qualidade utilizando a metodologia do Projeto Axiomático}

$\mathrm{U}$ m modelo de gestão sistêmica da qualidade deve possuir amplo domínio de abrangência onde seja possível a interação de agentes distintos que, em geral, possuem desejos, necessidades e interpretações diferentes sobre o papel da qualidade dentro de uma organização. Contudo, alguns aspectos são relevantes nesta proposta para que se caracterize o contexto em que se dá o desenvolvimento do modelo de gestão sistêmica da qualidade, que neste caso foi idealizado considerando aspectos inerentes aos sistemas de produção e apoio de uma organização industrial do segmento metal-mecânico. Obviamente, não se pode deixar de reconhecer que outros aspectos relevantes (tipo de produto, mercado, tipo de produção, etc.) são importantes na caracterização do contexto em que se dará o desenvolvimento deste modelo, inclusive pela necessidade de contemplar características específicas de certas organizações. No entanto, por se tratar de uma proposta exploratória de caráter sistêmico, este trabalho estará pautado em aspectos mais generalistas relativos à caracterização das organizações. Assim, dentro desta abordagem como ponto inicial foram caracterizados os agentes que interagiam com 
um modelo de gestão sistêmica de qualidade em uma organização, bem como descritos quais seriam seus atributos.

Dentro deste escopo foram definidos quatro grupos de agentes que interagem, direta ou indiretamente, com o modelo de gestão sistêmica da qualidade a nível de uma organização, sendo os mesmos: acionistas da organização; colaboradores internos da organização; consumidores dos produtos da organização e comunidade em que se insere a organização. Para o domínio de clientes deve-se então determinar quais seriam as principais necessidades ou desejos dos grupos de clientes em relação ao modelo de gestão sistêmica da qualidade, constituindo assim os atributos do cliente a serem considerados no desenvolvimento do modelo. A Tabela 2 indica os grupos de agentes e atributos do cliente propostos em relação ao modelo proposto de gestão sistêmica da qualidade.

O passo seguinte no projeto de um modelo de gestão sistêmica da qualidade é a determinação de um requisito funcional de mais alto nível da estrutura hierárquica no domínio funcional, pois toda a estruturação do modelo de gestão sistêmica da qualidade dependerá da escolha deste requisito funcional de mais alto nível, sendo os níveis inferiores desdobrados a partir deste requisito funcional escolhido. Para este modelo proposto foi definido o seguinte requisito funcional como o de mais alto nível na estrutura hierárquica:

FR1 : Maximizar o retorno sobre o investimento

A escolha deste requisito funcional como o de mais alto nível na estrutura hierárquica deu-se em função do contexto anteriormente descrito e considerado para o desenvolvimento deste modelo, ressaltando-se também como outros aspectos relevantes:

a) Tal requisito funcional representa um atributo de cliente relativo aos acionistas da organização, os quais constituem um grupo de agentes que interagem, talvez de uma forma mais secundária, com a gestão da qualidade de uma empresa. Assim, sob tal ponto de vista, a escolha deste FR1 também já representaria o atendimento das necessidades deste grupo de clientes da empresa.

b) A definição deste FR1 parte do pressuposto, dentro da lógica de economias capitalistas, que uma das principais finalidades das organizações com fins lucratícios é de remunerar adequadamente o capital investido pelos acionistas. Além disso, dento desta lógica, deve-se considerar que a partir do ponto em que fosse possível a uma empresa gerar lucros e atender primeiramente aos atributos de cliente do grupo de acionistas, criaria-se condições adequadas para a existência desta empresa. Originam-se assim as condições para que os atributos de clientes de outros grupos de agentes (colaboradores internos da organização, consumidores dos produtos da organização e comunidade em que a organização se insere) também pudessem ser contemplados à medida que se desse a decomposição em níveis inferiores da estrutura hierárquica.

Após a definição do requisito funcional de mais alto nível na estrutura hierárquica do domínio funcional, deve-se proceder ao mapeamento no domínio físico de quais poderiam ser os parâmetros de projeto que atendem ao FR1 a este nível hierárquico. Cabe ressaltar que o FR1 definido pode ser atendido por diferentes linhas de abordagem estratégica, as quais podem abranger desde uma ênfase preponderantemente financeira até uma ênfase pautada unicamente em aspectos operacionais de uma organização. Contudo, a proposta deste trabalho é buscar a conjugação de aspectos financeiros e operacionais, a partir da criação de um modelo de gestão sistêmica da qualidade que possa propiciar à empresa obter vantagens competitivas, direcionando-se pelo atendimento dos atributos de seus clientes. Neste contexto, o parâmetro de projeto determinado no domínio físico para atendimento de FR1 é dado por:

DP1 : Modelo de gestão sistêmica da qualidade 
Tabela 2 - Grupos de agentes e atributos do cliente relativos ao modelo proposto de gestão sistêmica da qualidade

\begin{tabular}{|c|c|}
\hline \multirow{2}{*}{ Grupo de Agentes } & Atributos do Cliente \\
\hline & Descrição \\
\hline - Acionistas da organização & - Maximizar o retorno sobre o investimento \\
\hline $\begin{array}{l}\text { - Colaboradores internos da } \\
\text { organização }\end{array}$ & $\begin{array}{l}\text { - Criar estratégias organizacionais para a prosperidade do negócio (nível } \\
\text { hierárquico de diretoria) } \\
\text { - Coordenar a aplicação de táticas para a melhoria de desempenho } \\
\text { organizacional (nível hierárquico de gerência) } \\
\text { - Implementar táticas para a melhoria de desempenho organizacional } \\
\text { (nível hierárquico de apoio e planejamento operacional) } \\
\text { - Executar as tarefas propostas para a melhoria do desempenho } \\
\text { organizacional (nível hierárquico de execução) } \\
\text { - Melhoria contínua no desempenho de suas atividades (todos níveis } \\
\text { hierárquicos da organização) }\end{array}$ \\
\hline $\begin{array}{l}\text { - Consumidor dos produtos da } \\
\text { organização }\end{array}$ & $\begin{array}{l}\text { - Adquirir produtos que contemplem necessidades de valor percebido } \\
\text { - Receber produtos segundo expectativas estabelecidas }\end{array}$ \\
\hline $\begin{array}{l}\text { - Comunidade em que a } \\
\text { organização se insere }\end{array}$ & - Coexistência harmoniosa com a organização \\
\hline
\end{tabular}

\subsection{Estabelecimento do $2^{\circ}$ nível da estrutura} hierárquica: decomposição de FR1 pelo processo de zig zag e determinação dos correspondentes parâmetros de projeto

Tendo definido o requisito funcional FR1 e o correspondente parâmetro de projeto DP1, os quais representam os mais altos níveis na estrutura hierárquica de domínios funcional e físico, respectivamente, a etapa seguinte dentro da abordagem do Axiomatic Design é conduzir o processo de zig zag, ou seja, retornar do domínio físico para o domínio funcional caso o parâmetro de projeto não possa ser implementado sem posteriores detalhamentos no projeto. Os níveis inferiores subseqüentes da estrutura hierárquica obedecem ao mesmo procedimento, ou seja, decomposição de FRs resultando em novos requisitos funcionais necessários à implementação de DPs que, por sua vez, determinarão novos parâmetros de projeto em níveis inferiores.

Para a decomposição de FR1 faz-se necessário preliminarmente algumas considerações com respeito ao conceito de retorno sobre o investimento. A denominada taxa de retorno sobre o investimento, comumente indicada pela sigla ROI (abreviatura da expressão return over investiment) foi desenvolvida e aplicada inicialmente no inicio do século XX na empresa Du Pont Company, como um importante indicador contábil que possibilitava uma avaliação do sucesso comercial de suas unidades operacionais e da organização como um todo (JOHNSON \& KAPLAN, 1987). Para a época representou uma importante inovação contábil, perdurando até os dias atuais como um indicador contábil de desempenho financeiro de uma empresa. Apesar do enfoque da taxa do ROI poder ser desdobrado em um indicador financeiro de grande utilização em análises contábeis quando considera o valor de vendas $(\mathrm{ROI}=$ Margem de Lucro x turnover do ativo), neste trabalho não foram considerados tais detalhamentos contábeis, tomando-se a taxa do ROI em sua forma original consolidada, adaptada com relação ao enfoque no sistema produtivo, sendo dessa forma caracterizada por:

$\mathrm{ROI}=($ Receita de venda de produto - Gastos na produção) / Investimento na produção 
Deve-se ressaltar que a consideração de investimento na produção visa enfocar aspectos relevantes para o desenvolvimento deste trabalho e que focalizem a questão na área produtiva de uma empresa, embora não possam ser descartados como importantes investimentos de uma empresa as áreas comerciais e de pós-venda, os quais não serão abordados dentro do escopo deste modelo proposto de gestão sistêmica da qualidade. Feitas tais considerações e dada a decomposição do requisito funcional FR1 (maximizar o retorno sobre o investimento), os requisitos funcionais determinados no $2^{\circ}$ nível da estrutura hierárquica do domínio funcional foram definidas como: FR11 (Aumentar a receita de venda de produtos), FR12 (Minimizar gastos na produção) e FR13 (Minimizar investimentos na produção). Procede-se agora a definição dos correspondentes parâmetros de projeto, os quais representam uma decomposição de DP1 (Modelo de gestão sistêmica da qualidade) no domínio físico, sendo para este caso definidos por: DP11 (Produtos que maximizam a satisfação do cliente), DP12 (Estabelecimento de um custo alvo do produto) e DP13 (Avaliação sistêmica do investimento na produção).

Os produtos que maximizam a satisfação do cliente (DP11) serão aqueles produtos que terão sua preferência de compra, de forma que segundo critérios pessoais o consumidor buscará maximizar a sua satisfação no ato da compra do produto. Assim, produtos que maximizam a satisfação do cliente (DP11) permitem aumentar a receita de vendas de produtos (FR11). Além disso, a partir do ponto em que se maximiza a satisfação do consumidor, tem-se menores alterações de produtos, menores necessidades promocionais, maior adequação e direcionamento dos recursos produtivos voltados para a satisfação do cliente. Esta situação também possibilita uma otimização na utilização da capacidade produtiva, propiciando uma adequação dos investimentos que serão feitos para atender uma demanda direcionada pelo mercado, atendendo assim ao consumidor no que ele deseja e no tempo em que necessita. Dessa forma, produtos que maximizam a satisfação do cliente (DP11) possibilitariam também minimizar gastos da produção (FR12) e investimentos na produção (FR13).

O estabelecimento de um custo alvo do produto (DP12) está condicionado ao preço que o mercado estaria disposto a pagar pelo produto, influindo assim em um parâmetro de inserção e concorrência do produto no mercado, ou seja, o preço alvo. Quanto menor for o preço alvo do produto comparativamente aos produtos concorrentes, maior poderá ser a vantagem competitiva do produto baseado no aspecto preço ao consumidor. Dessa maneira, o estabelecimento de um custo alvo do produto (DP12) possibilita minimizar gastos na produção (FR12) e minimizar investimentos na produção (FR13), já que um produto só será produzido a partir do ponto em que seu custo de produção seja menor ou igual ao custo alvo definido pelas condições do mercado.

A avaliação sistêmica do investimento na produção (DP13) esta pautada na premissa de otimização de investimentos visando obter um maior retorno financeiro para o capital investido, ou seja, para um dado capital investido busca-se conferir ao sistema produtivo flexibilidade tanto a nível de capacidade produtiva, como também a nível de diversidade do mix de produção. Dessa forma, dentro da visão proposta neste trabalho, a avaliação sistêmica do investimento na produção (DP13) possibilitaria minimizar investimentos na produção (FR13).

Tendo sido determinado o conjunto de FRs e DPs, deve-se determinar a matriz de projeto e verificar se a mesma observa o Axioma da Independência, sendo que para este caso teria-se a seguinte situação:

$$
\left\{\begin{array}{l}
\text { FR 11 } \\
\text { FR 12 } \\
\text { FR 13 }
\end{array}\right\}=\left[\begin{array}{lll}
\mathrm{X} & 0 & 0 \\
\mathrm{X} & \mathrm{X} & 0 \\
\mathrm{X} & \mathrm{X} & \mathrm{X}
\end{array}\right] \cdot\left\{\begin{array}{l}
\mathrm{DP} 11 \\
\mathrm{DP} 12 \\
\mathrm{DP} 13
\end{array}\right\} \quad \text { (matriz 1) }
$$

Observando-se que $X$ indica um forte relacionamento entre FR e DP, verifica-se neste caso 
tratar-se de um projeto decoupled caracterizado pela matriz triangular, o qual satisfaz o Axioma da Independência, caracterizando-se assim o segundo nível hierárquico do modelo proposto de gestão sistêmica da qualidade.

\subsection{Estabelecimento do $3^{\circ}$ nível da estrutura hierárquica: decomposição de FR11, FR12 e FR13 pelo processo de $z$ ig zag e determinação dos correspondentes parâmetros de projeto}

A partir do $2^{\circ}$ nível da estrutura hierárquica pode-se visualizar a formação de 03 ramos originados respectivamente por FR11, FR12 e FR13, sendo que cada ramo atende ao requisito funcional de mais alto nível (FR1). Cada ramo exercerá influências distintas no modelo de gestão sistêmica da qualidade (DP1), sendo por isso analisados de forma individualizada.

\section{a) Ramo de aumento da receita de vendas (FR11)}

A receita de vendas de um produto pode ser dada por:

$\begin{gathered}\text { Receita } \\ \text { de } \\ \text { Vendas }\end{gathered}=\begin{gathered}\text { Preço de } \\ \text { venda do } \\ \text { produto }\end{gathered} \quad x \quad \begin{gathered}\text { Volume de } \\ \text { produtos } \\ \text { vendidos }\end{gathered}$

De maneira generalizada, para um mix de $n$ produtos teria-se:

$$
\mathrm{RTV}=\sum_{\mathrm{i}=1}^{\mathrm{n}}(\mathrm{PPi} \times \mathrm{VPi}), \text { onde: }
$$

RTV : Receita total de vendas

PP : Preço de venda do produto

VP : Volume de venda do produto

A decomposição de FR11 (aumentar a receita de venda de produtos) deve ser feita considerando o parâmetro de projeto definido DP11 (produtos que maximizem a satisfação do cliente), os quais irão gerar a necessidade de decomposição posteriores. Em função da relação anteriormente estabelecida entre RTV, PP e VP, pode-se verificar que neste caso o aumento da receita de vendas dar-se-ia pelos seguintes requisitos funcionais: FR111 (Maximizar o preço em função do valor percebido do cliente) e FR112 (Aumentar a quantidade de produtos vendidos).

Tendo definido os requisitos funcionais FR111 e FR112 no domínio funcional, o passo seguinte é a caracterização dos correspondentes parâmetros de projeto (DPs) que satisfaçam tais requisitos funcionais, estabelecendo assim o $3^{\circ}$ nível hierárquico da estrutura de domínio físico, a qual é dada neste modelo proposto por: DP111 (Diferenciação do produto) e DP112 (Produtos com maior aceitação no mercado).

Para se maximizar o preço em função do valor percebido pelo cliente (FR111) deve-se estabelecer uma diferenciação do produto (DP111) a qual possibilitaria contemplar o conjunto de valores do cliente quando do ato da efetivação da compra do produto. Esta maximização teria um preço máximo aceitável que se daria em função do estabelecimento de relações entre aspectos tangíveis e intangíveis do conjunto de valores do cliente. A diferenciação do produto (DP111) também pode aumentar a quantidade de produtos vendidos (FR112) à medida que novos potenciais clientes possam se sentir positivamente influenciados pelos clientes cativos ou serem atraídos pelas estratégias de marketing adotadas.

Para se aumentar a quantidade de produtos vendidos (FR112) deve-se buscar implementar ações que façam com que os produtos tenham uma maior aceitação no mercado (DP112), ressaltando-se a premissa da livre concorrência de mercados, o que impediria ou minimizaria a formação de mercados oligopolistas.

Assim, a matriz de projeto para o conjunto de FRs e DPs definidos ao $3^{\circ}$ nível da estrutura hierárquica com relação ao ramo de aumento de receitas de vendas seria dado por:

$$
\left\{\begin{array}{l}
\mathrm{FR} 111 \\
\mathrm{FR} 112
\end{array}\right\}=\left[\begin{array}{ll}
\mathrm{X} & 0 \\
\mathrm{X} & \mathrm{X}
\end{array}\right] \cdot\left\{\begin{array}{l}
\mathrm{DP} 111 \\
\mathrm{DP} 112
\end{array}\right\} \quad(\text { matriz 2) }
$$


Caracteriza-se, dessa forma, uma matriz de projeto decoupled, satisfazendo o Axioma da Independência a este nível hierárquico do modelo proposto de gestão sistêmica da qualidade.

\section{b) Ramo de minimização de gastos na produção (FR12)}

Pode-se definir gastos como “... sacrifício financeiro com que a entidade arca para a obtenção de um produto ou serviço qualquer, sacrifício esse representado por entrega ou promessa de entrega de ativos (normalmente dinheiro)" (MARTINS, 1993). Os gastos podem ser classificados pela terminologia contábil como: investimento, custo, despesa, desembolso e perda. Contudo, apesar desta diferença terminológica, usualmente se adota como conceito de custos aqueles gastos relativos ao consumo na produção, ao passo que as despesas seriam aqueles gastos que se destinam às fases de administração, esforço de vendas e financiamento. Esse é um conceito extremamente amplo e que se aplica a todos os bens e serviços recebidos pela empresa.

$\mathrm{O}$ escopo proposto neste modelo restringiu-se ao foco na produção, definindo-se assim os seguintes requisitos funcionais desdobrados de FR12 (Minimizar gastos na produção): FR121 (Definir o custo alvo da produção) e FR122 (Estabelecer limites de despesas administrativas na produção).

Tendo definido tais requisitos funcionais no domínio funcional, os correspondentes parâmetros de projeto serão decompostos a partir de DP12 (Estabelecimento de um custo alvo do produto), originando assim o $3^{\circ}$ nível hierárquico da estrutura de domínio físico para o ramo de minimização de gastos na produção, o qual será composto por: DP121 (Enfoque baseado na gestão total de custos) e DP122 (Gerenciamento das despesas administrativas da produção).

$\mathrm{O}$ enfoque baseado na gestão total de custos (DP121) fundamenta-se na análise de custos sob um contexto mais amplo do enfoque meramente contábil-financeiro, incluindo elementos de análise estratégicas da empresa, direcionando a contabilidade gerencial para exercer um papel relevante ao êxito da empresa, considerando outros fatores relevantes à empresa e possibilitando uma alocação de custos mais acurada dentro do processo produtivo. Dentro desta contextualização do enfoque baseado na gestão total de custos deve-se então definir o custo alvo da produção (FR121).

$\mathrm{O}$ gerenciamento das despesas administrativas da produção (DP122) busca estabelecer um controle sobre possíveis reduções no patrimônio líquido da empresa, haja vista que a lógica contábil de abatimento de despesas se dá diretamente do Resultado, quando se adota procedimentos baseados em sistemas de custeio total com bases de rateio por absorção ou taxas (MARTINS, 1993). Dessa forma, o gerenciamento das despesas administrativas da produção (DP122) pode estabelecer bases para que a empresa crie políticas que visem estabelecer limites de despesas administrativas na produção (FR122), ressaltando que dentro do escopo proposto neste modelo não serão abordados os enfoques de despesas relativas ao esforço de vendas e financiamentos.

A matriz de projeto para o conjunto de FRs e DPs definidos no $3^{\circ}$ nível hierárquico com relação ao ramo de minimização de gastos na produção seria dado por:

$$
\left\{\begin{array}{l}
\text { FR121 } \\
\text { FR122 }
\end{array}\right\}=\left[\begin{array}{ll}
\mathrm{X} & 0 \\
0 & \mathrm{X}
\end{array}\right] \cdot\left\{\begin{array}{l}
\mathrm{DP} 121 \\
\mathrm{DP} 122
\end{array}\right\} \quad(\text { matriz 3) }
$$

Esta caracteriza-se como um matriz de um projeto uncoupled, a qual satisfaz o Axioma da Independência para este nível hierárquico do modelo proposto de gestão sistêmica da qualidade.

\section{c) Ramo de minimização de investimento na produção (FR13)}

Pode-se definir investimento como “... gasto ativado em função de sua vida útil ou de benefícios atribuíveis a futuro(s) período(s)..." 
(MARTINS, 1993). Assim, são denominados de investimentos todos os gastos que podem ser incorporados nos ativos da empresa para que se dê sua baixa ou amortização quando de sua venda, consumo, desaparecimento ou de sua desvalorização. Dessa forma, o requisito funcional FR13 (Minimizar investimentos na produção) pode ser decomposto no domínio funcional, considerando o correspondente parâmetro de projeto DP13 (Avaliação sistêmica do investimento na produção), definindo-se os seguintes requisitos funcionais de $3^{\circ}$ nível na estrutura hierárquica: FR131 (Avaliar investimentos em bens de capital), FR132 (Reduzir investimentos em matéria-prima e insumos) e FR133 (Avaliar investimentos em infraestrutura operacional).

Tendo-se definido os requisitos funcionais FR131, FR132 e FR133 no domínio funcional, parte-se para a definição dos correspondentes parâmetros de projeto que satisfaçam tais requisitos funcionais e que representem uma decomposição de DP13 (avaliação sistêmica do investimento na produção), estabelecendo assim o $3^{\circ}$ nível na estrutura hierárquica do domínio físico, o qual é dado por: DP131 (Ênfase na aquisição de equipamentos adaptáveis às alterações da demanda), DP132 (Ênfase na operação com baixos níveis de inventário) e DP133 (Ênfase na implantação de instalações e facilities adaptáveis à mudança).

A ênfase na aquisição de equipamentos adaptáveis às alterações da demanda (DP131), possibilita avaliar os investimentos em bens de capital (FR131), sob a ótica da aquisição de máquinas e equipamentos cuja capacidade produtiva estivesse dimensionada para atender a demanda do cliente, apresentando baixa complexidade na configuração e operação, reduzindo-se a necessidade de automação rígida incorporada e/ou de suporte aos bens de capital. A aquisição destes equipamentos adaptáveis às alterações da demanda também conduziria à necessidade de se avaliar os investimentos em infraestrutura operacional (FR133), devendo-se considerar estas perspectivas.
A ênfase na operação com baixos níveis de inventário (DP132) permitiria reduzir investimentos em matéria-prima e insumos (FR132), dada a operação dentro dos preceitos do just in time e estabelecimento de níveis mínimos operacionais de inventário (estoques de matériaprima, material em processo e produtos acabados). Também permitiria avaliar investimentos em infra-estrutura operacional (FR133), pois demandariam situações distintas de atividades de apoio à logística de gestão de materiais.

A ênfase na implantação de instalações e facilities adaptáveis à mudança (DP133), permitiria avaliar investimentos em infraestrutura operacional (FR133), considerando que as alterações de lay out fabril que viessem a ocorrer utilizassem a infra-estrutura operacional (instalações elétricas, redes de ar comprimido e água, sistemas de refrigeração, sistemas de coletas de resíduos, etc) já existentes.

Assim, a matriz de projeto para o conjunto de FRs e DPs definidos no $3^{\circ}$ nível da estrutura hierárquica com relação ao ramo de minimização de investimento na produção seria dada por:

$$
\left\{\begin{array}{l}
\text { FR 131 } \\
\text { FR 132 } \\
\text { FR 133 }
\end{array}\right\}=\left[\begin{array}{ccc}
X & 0 & 0 \\
0 & X & 0 \\
X & X & X
\end{array}\right] \cdot\left\{\begin{array}{l}
\text { DP 131 } \\
\text { DP 132 } \\
\text { DP 133 }
\end{array}\right\}
$$

(matriz 4)

Caracteriza-se assim uma matriz triangular de um projeto decoupled, o qual satisfaz o Axioma da Independência a este nível hierárquico do modelo proposto de gestão sistêmica da qualidade. Dessa forma, procedendo-se sistematicamente aos procedimentos de desdobramento e decomposição dos domínios funcionais e de projeto, é possível a estruturação dos denominados diagramas hierarquizados de requisitos funcionais (FRs) e parâmetros de projeto (DPs). Os diagramas de estruturas hierarquizadas de FRs e DPs são também conhecidos como "diagrama-árvore" de FR e DP, indicando a hierarquia estabelecida de FRs no domínio funcional e DPs no domínio físico, sendo que 


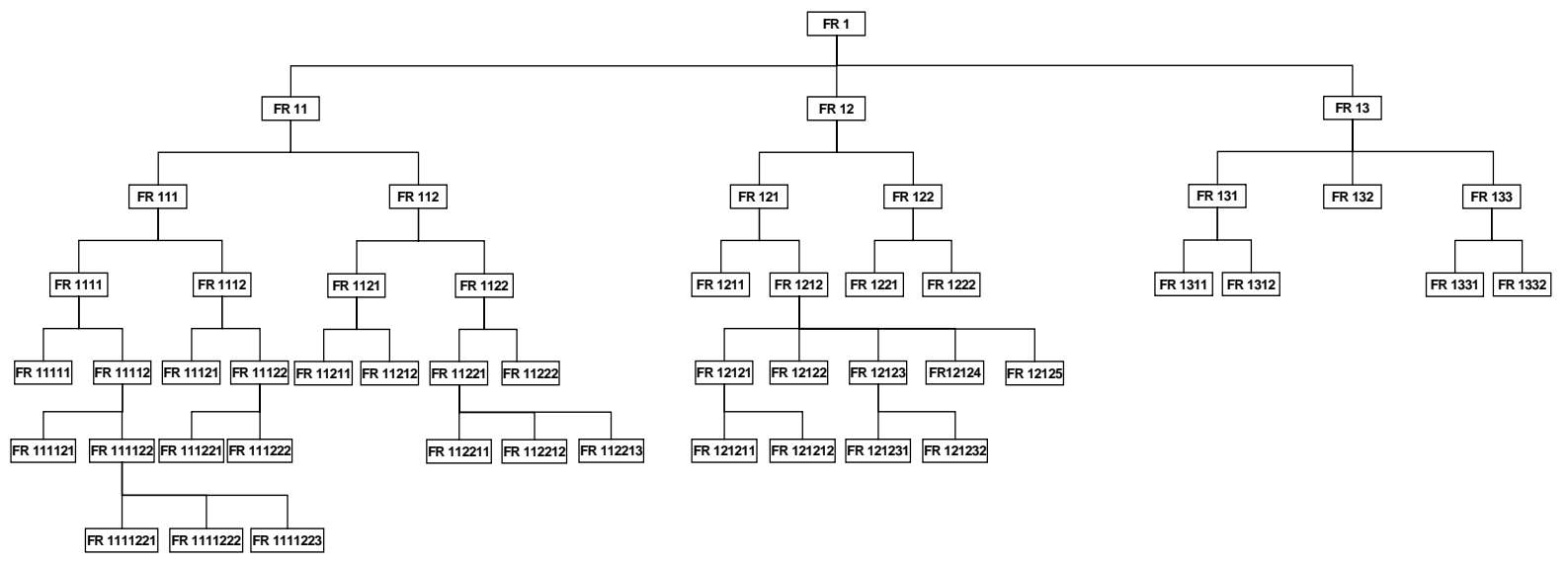

Figura 2 - Estrutura esquemática do "diagrama-árvore" de requisitos funcionais (FRs) propostos no modelo

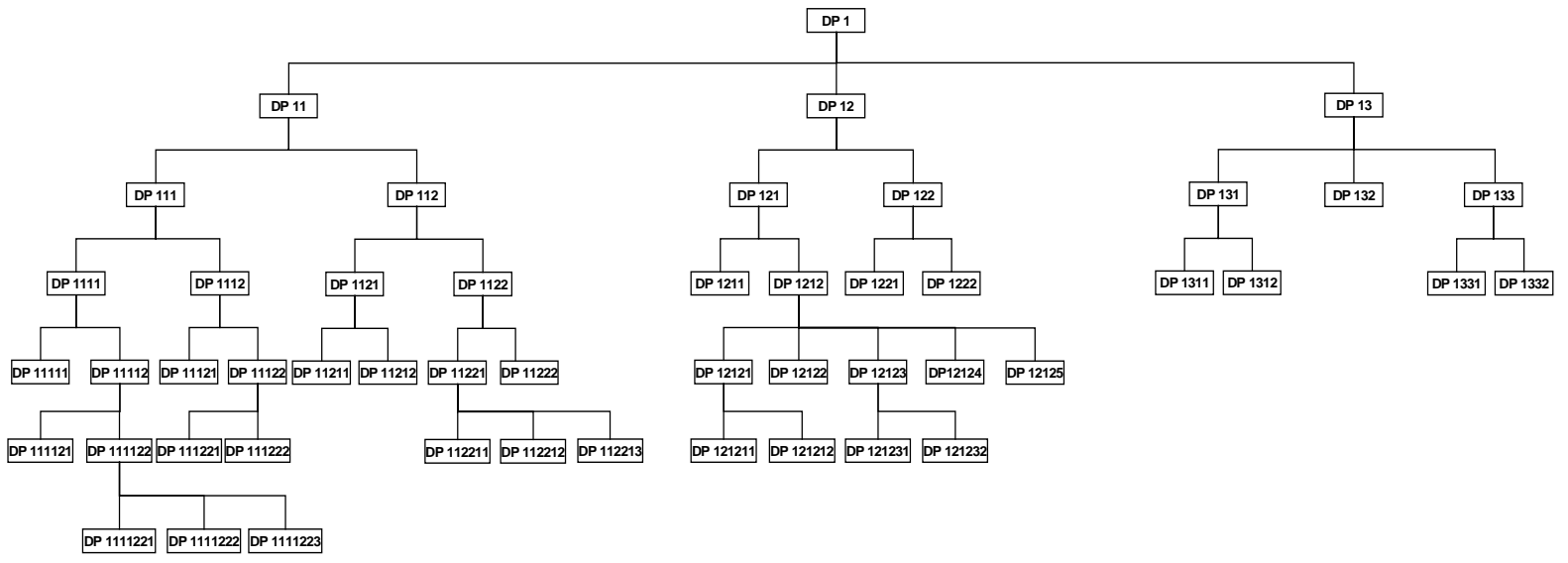

Figura 3 - Estrutura esquemática do “diagrama-árvore” de parâmetros de projeto (DPs) propostos no modelo

uma das principais finalidades do "diagramaárvore" está na simplificação e compreensão do projeto como um todo, indicando as relações entre FRs e DPs a cada nível hierárquico do "diagrama-árvore" (SUH, 1990). As Figuras 2 e 3 ilustram para o modelo de gestão sistêmica da qualidade desenvolvido as estruturas hierarquizadas de requisitos funcionais e parâmetros de projeto.

Além do desenvolvimento do "diagramaárvore" de FRs e DPs, para cada "ramo" das estruturas hierarquizadas foram obtidas as equações e matrizes de projeto, indicando se a relação estabelecida entre FRs e DPs definia uma matriz de projeto coupled, decoupled ou uncoupled. Dessa forma, através da aplicação dos princípios estabelecidos pela metodologia do Axiomatic Design foi desenvolvida a proposta do modelo de gestão sistêmica da qualidade, sendo na sua totalidade composta por 50 requisitos funcionais e 50 parâmetros de projeto, relacionados através de 21 matrizes de projeto e desdobrados em 7 níveis hierárquicos. A obtenção dos níveis hierárquicos se dá pelo processo de zig zag, fazendo-se a decomposição a partir dos níveis hierárquicos superiores $\mathrm{e}$ obtendo-se assim os níveis inferiores da estrutura. 


\section{Considerações Finais}

A abordagem axiomática utilizada no desenvolvimento deste trabalho mostrou viabilidade de aplicação, bem como um significativo potencial na estruturação de uma metodologia para construção e sistematização de um modelo de gestão da qualidade, que não deve estar unicamente pautado no pragmatismo das certificações de sistemas da qualidade, mas que possa também captar certas abstrações e complexidades relativas ao contexto em que se inserem as organizações. A caracterização dos atributos do cliente (CAs) e a definição dos requisitos funcionais buscando contemplar tais atributos do cliente, direciona para o que se pode denominar de "boas práticas" de projeto, além de contemplar aspectos tão citados e almejados por modelos de gestão da qualidade com enfoque no TQM (Total Quality Management), no sentido de estarem focados e direcionados pelos clientes.

Os relacionamentos estabelecidos entre parâmetros de projeto e requisitos funcionais, caracterizados pelas matrizes de projeto, indicam de maneira clara e convincente quais seriam as melhores escolhas de DPs para atender aos FRs. O desenvolvimento de estruturas hierárquicas nos domínios funcional e físico possibilitam a visão sistêmica do modelo, bem como as interações e relacionamentos estabelecidos. A análise das matrizes de projeto a cada nível hierárquico permitem uma melhor orientação na escolha do DP mais adequado no atendimento de determinado FR, considerando o contexto em que se dá a decomposição hierárquica dos domínios funcional e físico.

Cabe ressaltar também a possibilidade de outras observações que podem ser feitas considerando a análise do que se denomina de "matriz consolidada de projeto", a qual contempla de maneira conjunta as matrizes individuais estabelecidas a cada nível hierárquico, analisando influências diagonais e transversais que podem ocorrer no modelo quando se considera uma matriz consolidada de projeto triangular.
Este é um importante aspecto a ser analisado quando são considerados modelos complexos com mecanismos que se inter-relacionam, como é o caso de organizações empresariais. Outros desdobramentos de análise e diagnóstico não foram abordados neste trabalho, tais como o "diagrama de estrutura de módulo e junção" e a especificação de medidas de desempenho conjugadas ao planejamento estratégico considerando a abordagem do Balanced Scorecard (KAPLAN \& NORTON, 1992), pretendendo-se discuti-los em artigo futuro.

A proposta deste trabalho na construção e sistematização de um modelo de gestão da qualidade que tenha um enfoque sistêmico, não só contempla a tendência verificada na bibliografia mais recente da área, como também traz uma contribuição na discussão sobre como sistematizar a gestão da qualidade com ênfase no atendimento das necessidades dos clientes e desenvolvimento de vantagens competitivas para a empresa. $\mathrm{O}$ modelo proposto contempla a visão mais atual do conceito de cliente, classificando-os como internos ou externos à organização e enfocando segmentos fundamentais tais como: acionistas, consumidores de produtos, sociedade e colaboradores internos. Os relacionamentos estabelecidos entre os requisitos funcionais e parâmetros de projeto nas estruturas hierarquizadas permitem compreender e caracterizar qual a influência do DP sobre o FR, possibilitando uma contribuição na área da qualidade, no sentido de estruturar, hierarquizar e relacionar programas, ferramentas e técnicas da qualidade com aplicações setoriais e específicas dentro de uma visão sistêmica de qualidade organizacional.

Contudo, algumas dificuldades e possíveis limitações na aplicação deste modelo foram verificadas e merecem um aprofundamento de estudos futuros. A primeira dificuldade é que a construção de um modelo de gestão sistêmica da qualidade pressupõe um conhecimento não só de aspectos relativos à qualidade, como outros aspectos relevantes ao contexto em que se dá a compreensão do significado do termo sistêmico. 
Dessa forma, os modelos podem contemplar por desdobramentos dos FRs e DPs, aspectos mais desejáveis e importantes para uma organização dentro de um contexto estabelecido, necessitando portanto de uma visão direcionada por um comitê de política da qualidade que possa representar várias áreas da organização. No entanto, esta dificuldade inerente ao domínio e a operacionalização da gestão de conhecimentos e informações na organização não obscurecem o potencial do modelo que é o de possibilitar uma orientação sistematizada na implementação das soluções propostas. A segunda dificuldade refere-se, no caso do modelo proposto, de conjugar aspectos de caráter tangível (facilmente quantificáveis) com aspectos de caráter intangível (dificilmente quantificáveis) na estruturação do modelo, dificultando o estabelecimento de um procedimento meramente quantitativo na análise e avaliação de desempenho. Esta discussão não deve ter como foco neste caso a separação dos aspectos tangíveis e intangíveis, já que um modelo sistêmico deve contemplar tais dicotomias, mas sim procurar aperfeiçoamentos que possibilitem contemplar uma abordagem quantitativa e qualitativa na estruturação do modelo proposto de gestão sistêmica da qualidade.

\section{Referências Bibliográficas}

BADIRU, A.B. \& AYENI, B.J.: Quality and process improvement. London: Chapman \& Hall, Chap.1: “The Quality Revolution”, p.1-35, 1994.

JOHNSON, H.T. \& KAPLAN, R.S.: Relevance lost - The rise and fall of management accounting. Harvard Business School Press, 269p., 1987.

KAPLAN, R.S. \& NORTON, D.P.: "The balanced scorecard - measures that drive performance", Harvard Business Review, January-February, p.71-79, 1992.

KIM, K.Y. \& CHANG, D.R.: "Global quality management: a research focus", Decision Sciences, v.26, n.5, p.561-568, September/October, 1995.

LAKHE, R.R. \& MOHANTY, R.P.: "Understanding TQM", Production Planning \& Control, v.5, n.5, p.426-441, 1994.

MARTINS, E.: Contabilidade de Custos. São Paulo: Editora Atlas, 311p., 1993.
MOHANTY, R.P.: “TQM: some issues for deliberation", Production Planning \& Control, v.8, n.1, p.10-13, 1997.

OAKLAND, J.S.: Gerenciamento da Qualidade Total - TQM. São Paulo: Nobel, p.31-49, 1994.

POWELL, T.C.: “Total Quality Management as competitive advantage: a review and empirical study", Strategic Management Journal, v.16, p.15-37, 1995.

SASHKIN, M. \& KISER, K.J.: Gestão da qualidade total na prática. Rio de Janeiro: Editora Campus, p.23-37, 1994.

SUH, N.P.: "Designing-in of quality through axiomatic design", IEEE Transactions on Reliability, p.256-264, v.44, n.2, June, 1995.

SUH, N.P.: The principles of design. New York: Oxford University Press, 401p., 1990. 


\title{
FROM THE TQM (TOTAL QUALITY MANAGEMENT) APPROACH TO GQM (GLOBAL QUALITY MANAGEMENT): THE INSERTION AND UTILIZATION OF THE AXIOMATIC DESIGN METHODOLOGY IN THE DEVELOPMENT OF A QUALITY SYSTEMIC MANAGEMENT MODEL
}

\begin{abstract}
In the past the word quality has been synonymous of success or failure in many firms, and this situation is a result of how these firms understand the quality concepts. The development of these are responsible for a kind of quality that has simply last its original means concerning products and processes control, in order to develop a systemic approach of quality management in the whole organization. The objective of this paper is to present a proposal of a quality systemic management model taking into account the firm's customer wants and characteristics. For the development of this work the Axiomatic Design approach is used, which is established in function of axioms, corollaries and theorems, with the objective of to improve "good practices of design" in the construction of the quality systemic management model.
\end{abstract}

Keywords: quality management, systemic approach, axiomatic design, quality systemic management. 